\title{
Dielectric multilayer angular filters for coupling LEDs to thin light guides
}

\author{
Cong MU ${ }^{\mathrm{a}}$, Hugo J. Cornelissen ${ }^{\mathrm{b}}$, Florian Bociort ${ }^{\mathrm{a}}$, Thomas Liebig ${ }^{\mathrm{a}}$ \\ aptics Research Group, Delft University of Technology, the Netherlands; \\ ${ }^{b}$ Philips Research Europe, High Tech Campus 34, 5656AE Eindhoven, The Netherlands
}

\begin{abstract}
A new approach is described to couple light from high-power blue LEDs $\left(1 \mathrm{x} 1 \mathrm{~mm}^{2}\right)$ into a thin large area light guide using a dielectric interference multilayer as an angular filter. The goal is to achieve large area luminaires such as backlight systems. The method overcomes the drawback of structuring holes or recessions in light guides when using a system with side-emitting LEDs. Several new LED-masking filters have been designed to improve the polarization dependency and coupling efficiency from a previous design that used a stack of inorganic bi-layers. Firstly, a polymeric multilayer manages to increase the coupling efficiency from $52 \%$ to $69 \%$. Secondly, a birefringent multilayer filter enables to fully suppress the Brewster's angle effect and to realize identical behaviour for both s- and p- polarized light at large angles. Thirdly, by adding layers of a third inorganic material to the original bi-layer stack with refractive index in between the other two, the transmittance equality is improved and the coupling efficiency can reach up to $63 \%$.

A novel angular filter is proposed for a large area planar collimating luminaire. A planar collimator of $2 \times 18^{\circ}$ cone angle with scalable and simplified structure is demonstrated. It has a thickness of only $5 \mathrm{~mm}$. Compact collimators and planar collimators have been analyzed with the ray tracing software LightTools. Measurements on this collimator filter and optical designs match well with the predicted performance.
\end{abstract}

A new approach to thin film filter design, the so called saddle-point method of global optimization was explored and showed promising initial results. A 40-layer collimator filter with a $30 \%$ lower merit function was obtained and is discussed.

Keywords: LED coupling, multilayer, collimator, LCD backlight, polymeric multilayer, birefringent polymer

\section{INTRODUCTION}

As the performance of Light Emitting Diodes (LEDs) keeps progressing to higher brightness, higher efficiency and a decreasing price per lumen, LEDs are rapidly taking the place of the conventional light sources such as fluorescent tubes and incandescent lamps in many applications [1]. One of the most significant applications is to couple LED light into a thin large area light guide to achieve a uniform backlight system for a Liquid Crystal Display (LCD). Due to the phenomenon of total internal reflection (TIR), light that travels with angles larger than the critical angle will keep propagating and be guided in the thin light guide. Eventually the light is extracted by micro-optics structures to illuminate the LC panel. The conventional side-emitting LEDs system requires complicated optical structures including recessions or holes and a thick light guide around $6 \mathrm{~mm}$. The combination of the LEDs and those structures requires precise alignment, which increases the manufacturing costs [2].

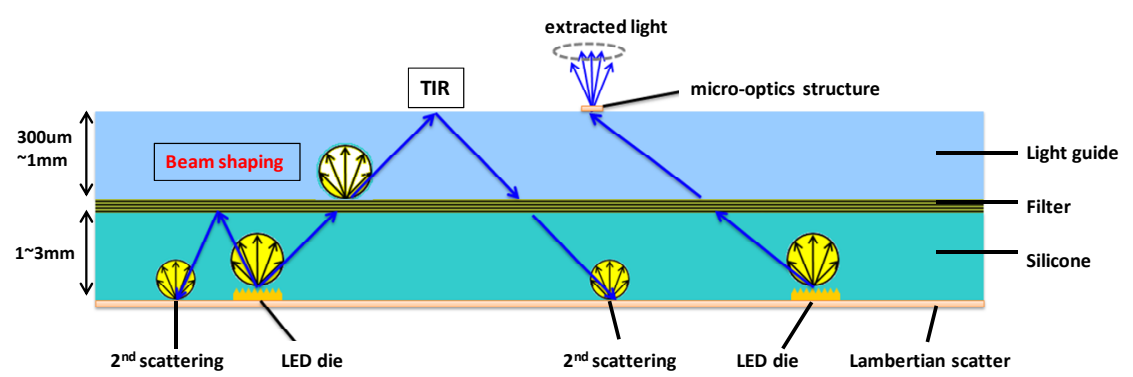

Fig. 1: Optical configuration of LED coupling into thin light guide using LED masking filter

Illumination Optics II, edited by Tina E. Kidger, Stuart David, Proc. of SPIE Vol. 8170, 81700 I

(C) 2011 SPIE · CCC code: $0277-786 X / 11 / \$ 18 \cdot$ doi: 10.1117/12.898156 
Our novel developed method is to realize LED coupling by using a dielectric interference multilayer as an angular filter. The filter will act as a mask to change the Lambertian light distribution into a specific output beam shape according to the specifications regarding angle, wavelength, and manufacturing. Multiple Luxeon Rebel bare LED dies, high power blue light sources with a central emission peak at $460 \mathrm{~nm}$, are directly positioned beneath the light guide and optically in contact with the filter, indicated in Fig. 1. The purpose of the interference multilayer is to only transmit light with incident angle larger than the critical angle and to obtain light guidance. Light with smaller angles is reflected back to the silicone rough bottom and recycled by the $2^{\text {nd }}$ Lambertian scattering. The LED-masking filter would ideally have the same performance for both polarization states.

\section{ANGULAR FILTER DESIGN}

As previously described [3], a LED-masking filter consisting of 54 layers of $\mathrm{Nb}_{2} \mathrm{O}_{5}\left(\mathrm{n}_{\mathrm{H}}=2.46\right)$ and $\mathrm{SiO}_{2}\left(\mathrm{n}_{\mathrm{L}}=1.46\right)$ was designed and optimized using the thin film optical design software Essential Macleod. As shown in Fig. 3, the transmittance performance at the peak wavelength of $460 \mathrm{~nm}$ exhibits a large difference between s- and p- polarized light, whereby the output coupling efficiency is reduced. This polarization dependency stems from the Brewster angle effect at oblique incidence, depending on the unequal optical admittances for s- and p- polarized light, illustrated in Fig. 2. The transmittance difference for both polarization states is proportional to the refractive index ratio of the materials. In most cases, our LED source emits un-polarized light. Concerning the total coupling efficiency, improvement of polarization equality is needed. Two possible solutions are selected. One is to use materials with lower index ratio for which we propose polymeric instead of the inorganic materials bi-layers. The other one is to add a third material with refractive index in between the previous two, which results in lower index ratios.

\subsection{Polymeric Multilayer Angular Filter}

The polymeric multilayer is constructed by multiple bi-layer stacks, whereby each stack consists of $m$ times of repeated unit cell (HL) ${ }^{\mathrm{m}}$, as shown in Fig. 4(a). H and L represent the quarter-wave layer of the high and low index materials respectively. According to references [4] and [5], for the polymeric material preferably PMMA $\left(\mathrm{n}_{\mathrm{L}}=1.49\right)$ and PEN $\left(\mathrm{n}_{\mathrm{H}}=\right.$ 1.75) are used to achieve a lower index ratio of 1.17, compared with the previous of 1.68. The HL layer for each stack does not have to be exactly a quarter wavelength thick. Essentially, by proper thickness tuning, each stack enables to reflect light of a different color spectrum. Consequently, the combination of bi-layer stacks with consecutive reflection bands is capable to broaden the overall stop bandwidth, as seen in Fig. 4(b). This broader band filter can effectively cut off the blue LED light with small incident angle. As the transmittance spectrum will shift to shorter wavelength with increasing incident angles, when arriving at the critical angle, the filter begins to transmit LED light and keeps doing so until $90^{\circ}$.

An isotropic polymeric multilayer LED-masking angular filter made of PEN and PMMA was designed in Essential Macleod. The filter was designed with seven bi-layer stacks and has a total thickness of $55.1 \mu \mathrm{m}$. The transmittance regarding to incident angle at the central peak at $460 \mathrm{~nm}$ is shown in Fig. 5(a) for both polarization states. The s- and pangular edge difference is minimized to $7^{\circ}$ compared with the previous $17^{\circ}$, which enhances the coupling efficiency.

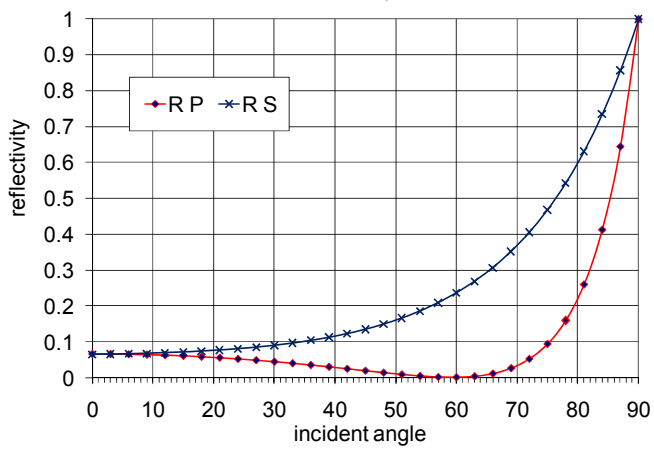

Fig. 2: Reflectance of $\mathrm{p}$ - and s-polarized light for various incident angle in red and blue at the interface $\mathrm{n}_{\mathrm{L}}=1.46$ to $\mathrm{n}_{\mathrm{H}}=2.46$. The Brewster angle is $59^{\circ}$.

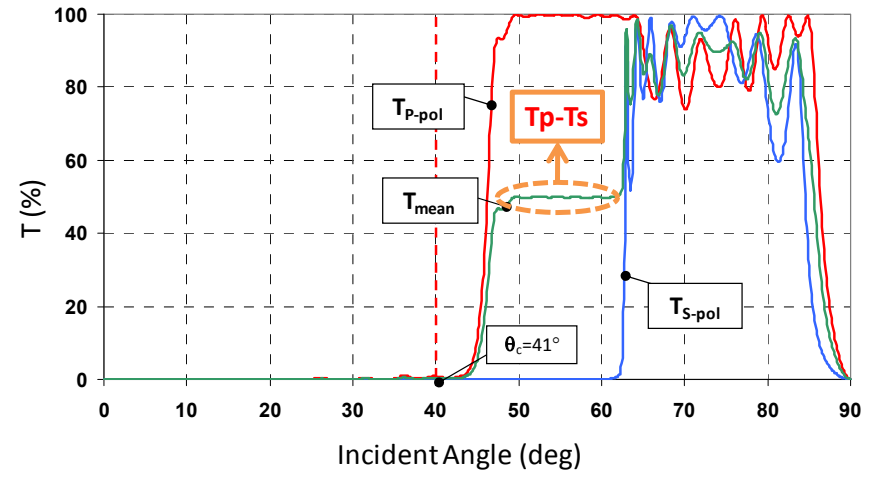

Fig. 3: Transmittance $\left(\theta_{\text {int }}\right)$ at the central wavelength of $460 \mathrm{~nm}$ for the HL LED-masking filter from ref.[3], red: p-polarized, blue: spolarized, green: mean. 


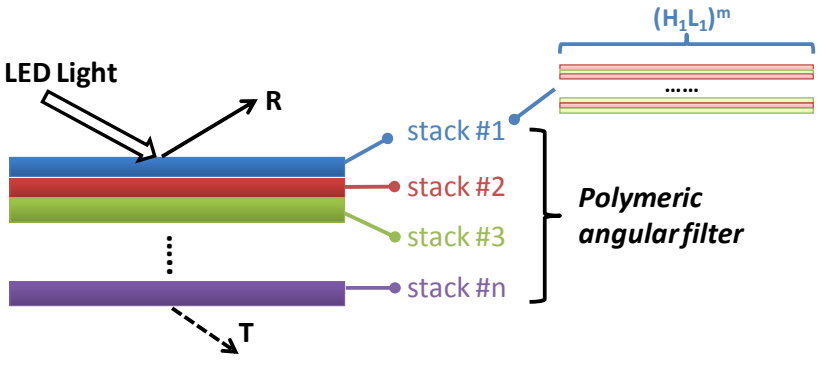

Fig. 4(a): The structure illustration for the polymeric multilayer angular filter made of bi-layer stacks

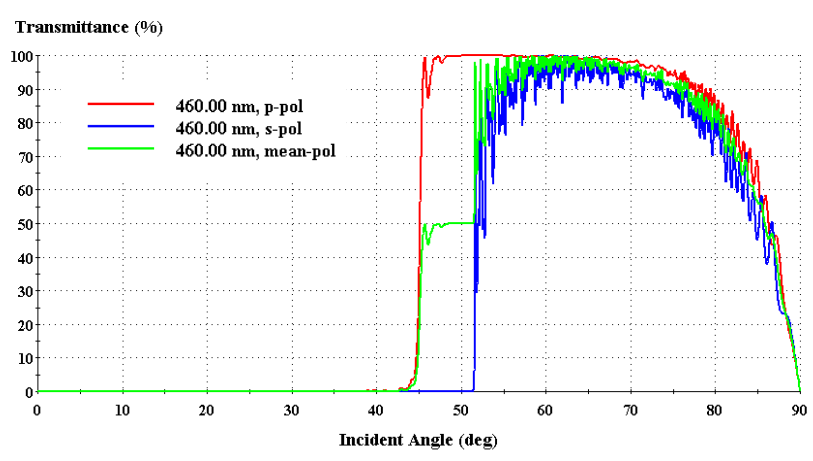

Fig. 5(a): Transmittance $\left(\theta_{\text {int }}\right)$ at the central wavelength of $460 \mathrm{~nm}$ of isotropic polymeric LED-masking filter

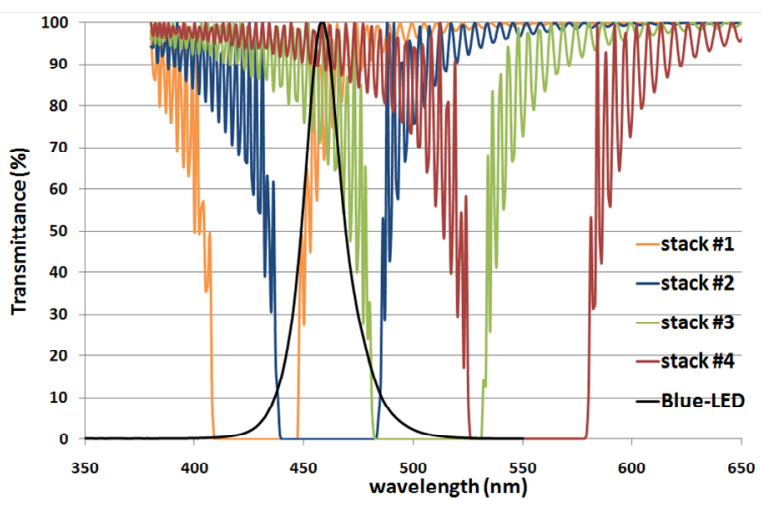

Fig. 4(b): Transmission spectra of 4 bi-layer stacks. The black curve shows the LED spectrum.

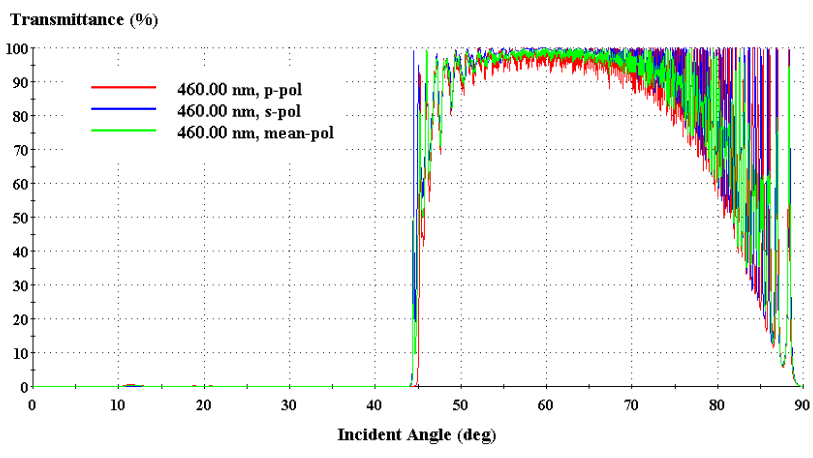

Fig. 5(b): Transmittance $\left(\theta_{\text {int }}\right)$ at the central wavelength of $460 \mathrm{~nm}$ of birefringent polymeric LED-masking filter

An elimination of the polarization dependency was achieved by using a birefringent polymeric multilayer to arbitrary control the Brewster angle as described in Ref. 6, where the reflectance of p-polarized light increases identically with spolarized light. This birefringent polymeric LED-masking filter was designed with the combination of two birefringent polymeric materials with a z-index mismatch. One possible combination could be $\mathrm{n}_{1 \mathrm{x}}=1.65, \mathrm{n}_{1 \mathrm{y}}=1.50, \mathrm{n}_{1 \mathrm{z}}=1.75 ; \mathrm{n}_{2 \mathrm{x}}=1.75$, $\mathrm{n}_{2 \mathrm{y}}=1.75, \mathrm{n}_{2 \mathrm{z}}=1.50$. As seen from Fig. 5(b), the transmittance curve at $460 \mathrm{~nm}$ shows an angular edge difference of less than $1^{\circ}$. This filter not only perfectly achieves polarization equality at large angles, but also blocks the light at small incident angles. The decrease of the mean transmittance from $80^{\circ}$ to $90^{\circ}$ results from the large number of polymeric layers in the combined stacks, in total approximately 700. Such a birefringent polymeric angular filter can possibly be manufactured with the technology that is described in Ref. 6.

\subsection{Optimization on HML, three material filter}

The high refractive index ratio of $\mathrm{Nb}_{2} \mathrm{O}_{5}(\mathrm{H})$ and $\mathrm{SiO}_{2}(\mathrm{~L})$ leads to the wide stop band and the transmittance gap between both polarization states. Using a third intermediate index material (M) allows for lower index ratio of $n_{H} / n_{M}$ and $n_{M} / n_{L}$, and additionally can help to suppress the higher order harmonics of p-polarized light at large incident angle. The added third material also provides more degrees of freedom for filter optimization and better chance to find a good result. Practically, we kept using the previous two high and low materials and selected an intermediate material $\mathrm{Y}_{2} \mathrm{O}_{3}$, with refractive index of 1.80 at $460 \mathrm{~nm}$. In Essential Macleod, the transmittance of s- and p- polarized light for various wavelength and incident angles was optimized. Weights and targets were set individually corresponding to the LED spectrum with inhomogeneous incident angle ranges. A fully automatic optimization process does not lead to satisfactory results. Instead, modification of the target settings and weights are required during the optimization process. The final filter performance of the HML LED-masking filter is illustrated in Fig. 6, with a total physical thickness of 6.94um. The 


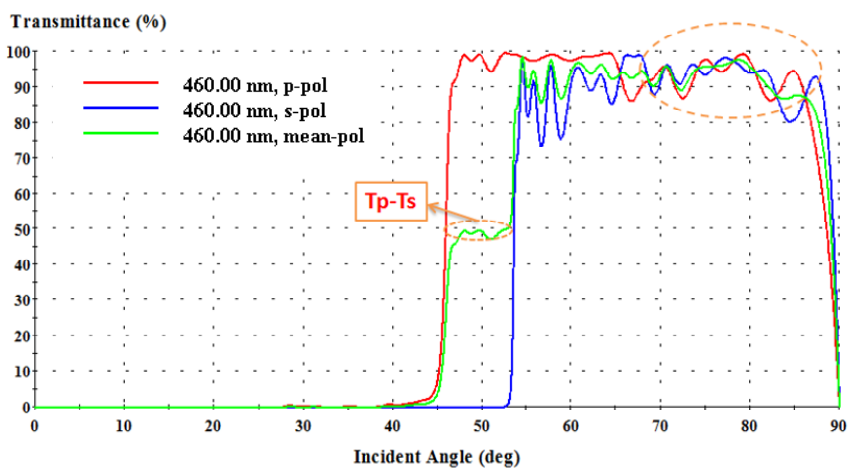

Fig. 6: Transmittance $\left(\theta_{\text {int }}\right)$ at central wavelength $460 \mathrm{~nm}$ of HML LED-masking filter

transmittance presented at $460 \mathrm{~nm}$ gives a smaller angular band edge difference of about $7^{\circ}$, and moreover effectively eliminates the oscillation ripples at larger incident angles of the previous design. This demonstrates the great potential of the three-material multilayer to create a satisfying angular filter design.

\section{COMPACT COLLIMATOR}

Based on the method of multilayer angular filter coupling, a compact collimator with a wedge-shaped reflective surface of $26^{\circ}$ was designed and prototyped [7]. The angular filter is deposited on top of a $1 \times 1 \mathrm{~mm}^{2}$ thin glass sheet which is attached on the Rebel Blue LED bare die. The wedge-shaped light guide is made of PMMA and the outside reflective coating is made of aluminum. Light passing the filter will hit the top surface of the light guide where it undergoes total internal reflection. Then the light reflects from the Al-coating surface and it is emitted with a small cone angle, as shown in Fig. 7(a). The calculated far field angular light distributions with $\mathrm{HL}$ filter $\left(\mathrm{Nb}_{2} \mathrm{O}_{5}\right.$ and $\left.\mathrm{SiO}_{2}\right)$, $\mathrm{HML}$ (three-material multilayer) and polymeric multilayer filter LED-masking filter are shown in green, red and blue respectively in Fig. 7(c). Optical ray tracing simulation is carried out in an identical LightTools compact collimator model which already matched well with the measurement result [7]. The output intensity along the normal has been greatly increased. The coupling efficiency has been improved from $58 \%$ to $70 \%$ for the polymeric LED-masking filter and to $66 \%$ for the HML filter. This verified that the new designed filters succeeded to increase the total output energy by having polarization equality.

\section{LARGE AREA PLANAR COLLIMATOR}

A simple large area planar collimator was designed by using another novel multilayer angular filter mounted on top of a $0.7 \mathrm{~mm}$ thick EK2 glass plate and exposed to the air, shown in Fig. 8. The LED sources are immersed at the bottom of the silicone rubber. The Lambertian distribution of the bare LED is changed into a collimated beam output after the filter. This overcomes the complicated lightguide shape and the precise alignment when combined with the LEDs. Light of

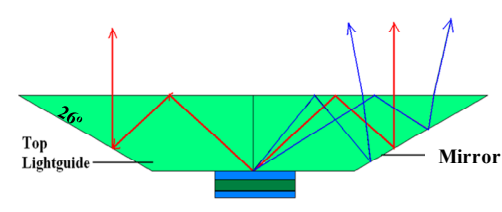

(a)

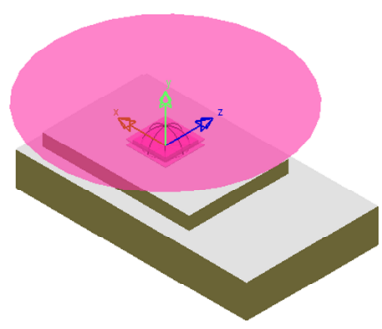

(b)

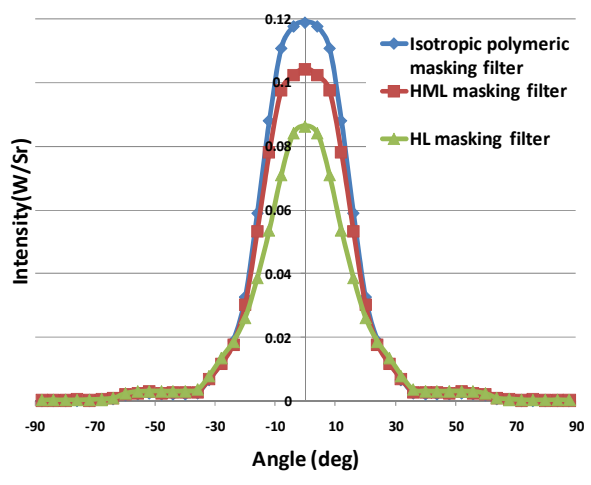

(c)

Fig. 7: (a) The working principle of the compact collimator; (b) Compact collimator model in LightTools; (c) Calculated far field angular distribution of the compact collimator with three different filters. 


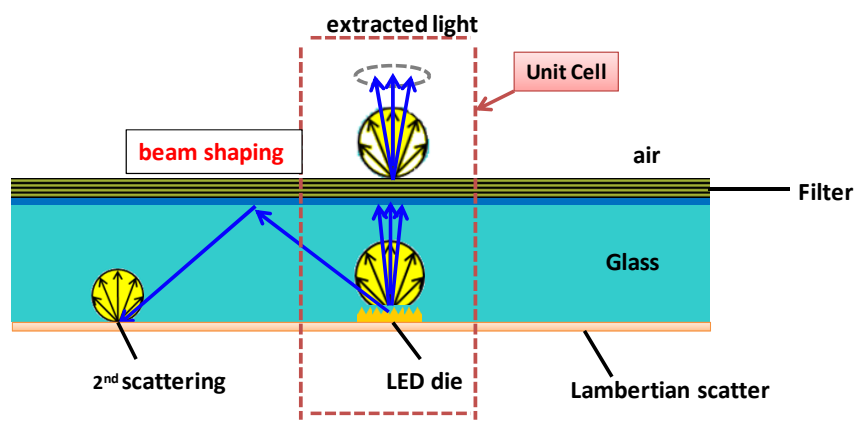

Fig. 8(a): The new optical configuration of a large area planar collimator

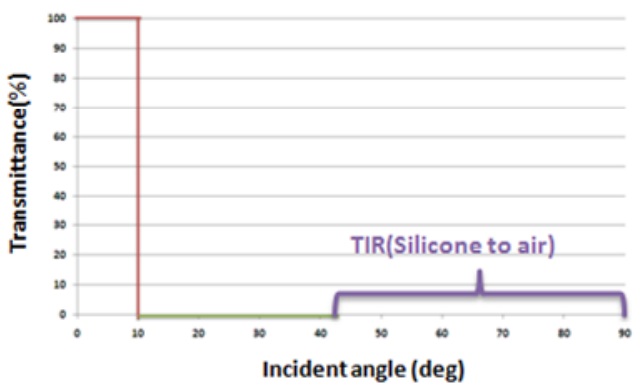

Fig. 8(b): Ideal transmittance as function of incident angle within the whole LED spectrum

small incident angles is transmitted by the filter, while light at large angles is reflected back and recycled by the $98 \%$ highly reflective white paint at the silicone bottom. The filter lies between the glass and the air. Light emitted larger than the critical angle consequently suffers TIR and is reflected back. The ideal filter performance and target settings are $\mathrm{T}_{\mathrm{p} 1}=\mathrm{T}_{\mathrm{s} 1}=100 \%$, for $\theta=\left[0^{\circ}: 1^{\circ}: 8^{\circ}\right]$ and $\mathrm{T}_{\mathrm{p} 2}=\mathrm{T}_{\mathrm{s} 2}=0 \%$, for $\theta=\left[10^{\circ}: 1^{\circ}: 42^{\circ}\right]$. A Nichia NS2B095AT blue medium power LED (central wavelength $468 \mathrm{~nm}$, FWHM of $25 \mathrm{~nm}$ ) is the selected light source.

\subsection{Collimator filter}

The collimator filter was designed with the available materials $\mathrm{SiO}_{2}$ and $\mathrm{Ta}_{2} \mathrm{O}_{5}$. The initial filter design and performance optimization were achieved in Essential Macleod with the above target settings. The final filter is a 34-layer, 2.69umthick HL multilayer film, with the p- and s- polarized angular transmittance at the peak wavelength of 468nm in Fig. 9(a). Transmitted light smaller than $10^{\circ}$ is maximized and good reflection is obtained from $18^{\circ}$ to the critical angle. Highly directional light output cannot be achieved over the whole LED spectrum. Some of the long wavelength part of the LED spectrum is reflected instead of transmitted at small angles and hence will not emerge from the collimator. Instead, it leaks out at large output angles of around $80^{\circ}$, indicated in Fig. 9(b) where for a wavelength of $481 \mathrm{~nm}$ a transmission peak is observed at angle close to the critical angle.

\subsection{Saddle point global optimization method}

Next to the classical design process, a new automatic approach for global optimization was applied to design the collimator filter, using an algorithm developed at TU-Delft referred to as Netmin. Netmin is a saddle point method, initially developed to investigate network structures in optical design [8], which are formed by the entirety of all links between local minima and saddle points of the merit function landscape. It has been applied to mirror-based objectives for EUV lithography [9], finally resulting in a new and very well performing system design. Also, the network structure of the double Gauss lens system has been revealed [10], delivering not only the best system configuration for the concrete design task, but also the full diversity of alternative configurations. These provide excellent starting designs for local optimization when other design requirements have to be met.

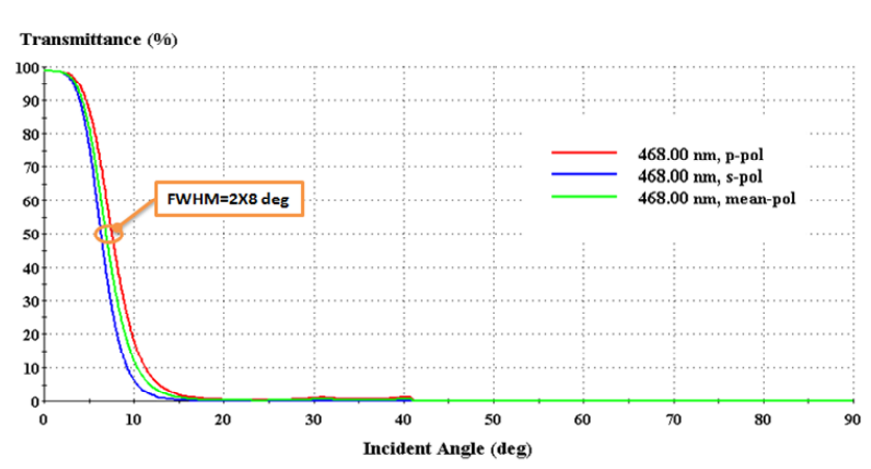

Fig. 9(a): Transmittance $\left(\theta_{\text {int }}\right)$ at the central wavelength of $468 \mathrm{~nm}$ of the collimator filter, showing a FWHM of $16^{\circ}$

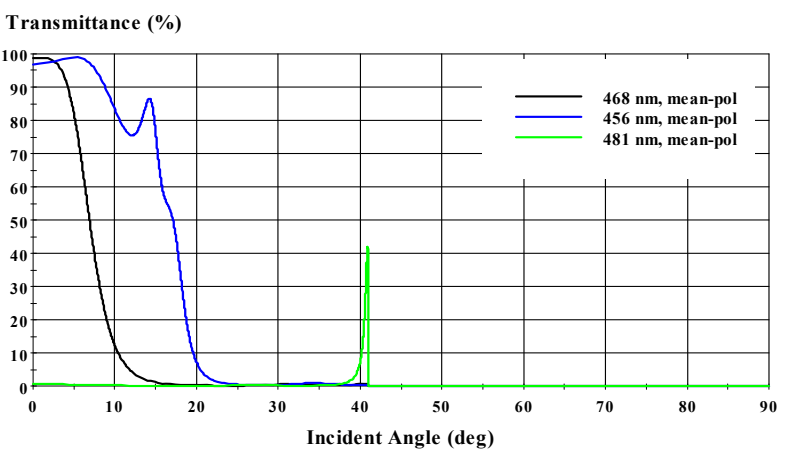

Fig. 9(b): Transmittance $\left(\theta_{\text {int }}\right)$ at the main three wavelengths (central, $\pm \mathrm{FWHM} / 2$ ) of the Blue LED spectrum 


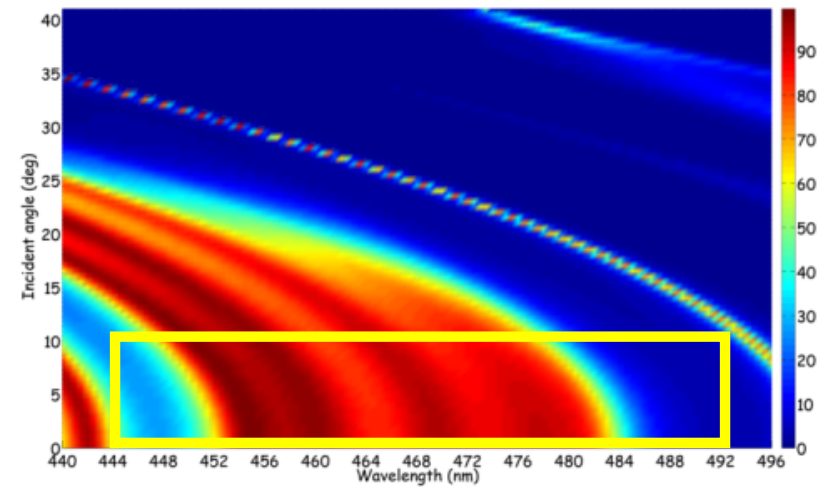

(a) s-polarization

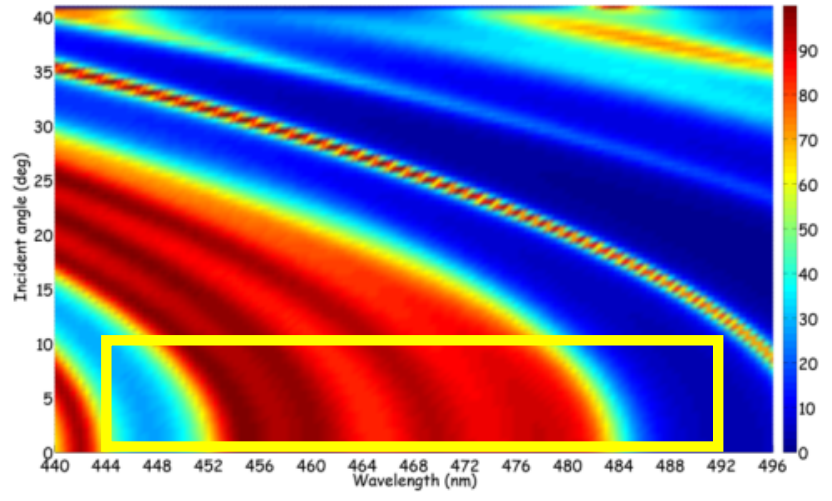

(b) p-polarization

Fig. 10: Transmittance (\%) of the best performing 40 layer collimator filter vs. wavelength and incident angle. The ideal filter would be fully red inside the yellow rectangle and blue elsewhere.

To start the global optimization an initial system design was chosen, representing a 40 layer bilayer thin film system of structure air $\left|(\mathrm{HL})^{20}\right|$ glass, using the same material and properties as mentioned for the collimator filter design. In order to reduce the numerical complexity every 10 layers were synthesized into 4 independent groups of strict $\mathrm{AB}$ periodicity, being described according to air $\left(\mathrm{H}_{1} \mathrm{~L}_{2}\right)^{\mathrm{m}}\left(\mathrm{H}_{3} \mathrm{~L}_{4}\right)^{\mathrm{m}}\left(\mathrm{H}_{5} \mathrm{~L}_{6}\right)^{\mathrm{m}}\left(\mathrm{H}_{7} \mathrm{~L}_{8}\right)^{\mathrm{m}} \mid$ glass with $\mathrm{m}=5$. Without prior-knowledge, every layer was initiated with a quarter wave optical thickness (qwot) at the center wavelength. This system was then optimized using a merit function with unitary weights over the spectral range, resulting in a local minimum. From this local minimum the connected saddle points in the merit function landscape were detected. Local optimization of the points close to the saddle points ends with new local minima found, closing one loop of iteration. The iterations ran automatically, only restricted by a thickness constraint of 4.8 qwot for each layer. In total, more than 3000 local minima have been obtained by this method, spanning the entire 8-parameter merit-function "landscape", each of them being different from any other minimum by an Euclidean distance of at least $5 \mathrm{~nm}$.

In order to solve the practical design problem, the synthesized groups were expanded to individual layers thereafter and a selection of local minima was used as starting values for optimization with Essential Macleod. Fig. 10 shows the transmittance characteristics of the best performing minimum. It exhibits a merit function value of about $30 \%$ less than the design obtained by the classical design process. However, the manual design was restricted to a total of 34 layers, while those evaluated by Netmin consist of 40 layers, according to the initialization of the search process. It was expected, that the total number of layers of the found minima would be reduced at the end of the design process due to manufacturing restrictions. With the investigated local minima this was not the case.

\subsection{Fabrication and Measurement}

The 34-layer collimator filter was deposited on a $0.7 \mathrm{~mm}$-thick EK2 glass of square size of $50 \times 50 \mathrm{~mm}^{2}$ by the thin film manufacturing facilities of Philips Innovation Services. The filter transmittance spectrum for various incident angles was measured by using a modified Perkin Elmer Spectrophotometer Lambda800 [7]. Comparison between the measured results in experiment and the calculated result from Essential Macleod were performed for incidence angles between $0^{\circ}$ to $40^{\circ}$ in glass and plotted in Fig. 11. It can clearly be seen that the measured result is in good agreement with the averaged calculated one, except for minor inequality from $0^{\circ}$ to $10^{\circ}$, which can be explained by the angular divergence effect of the measurement beam. From $0^{\circ}$ to $20^{\circ}$, within the FWHM of the LED spectrum, the light is highly transmitted, while from $20^{\circ}$ to $40^{\circ}$, LED light experiences a low mean transmittance. 


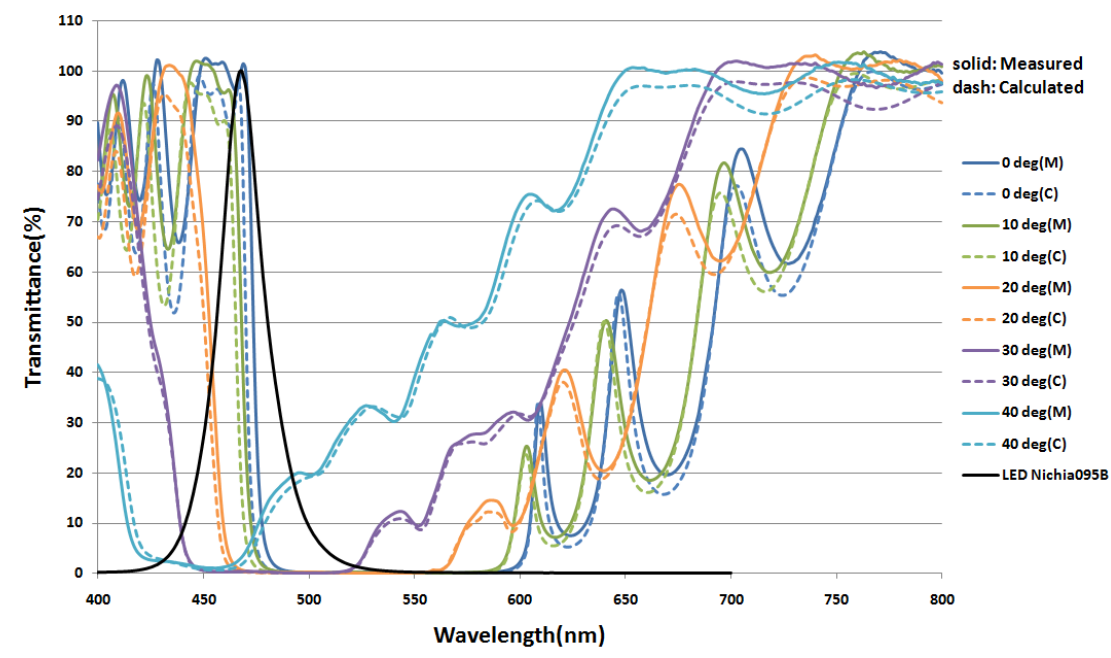

Fig. 11: Measured and calculated mean transmittance spectra at small incident angles. Angles are in glass. Solid lines refer to measured value and dashed lines refer to the calculated value. The black curve is the blue LED spectrum.

\subsection{Large Area Planar Collimator Sample}

The innovative large area planar collimating luminaire of Fig. 8 was modeled using LightTools. The design was simplified into a single unit cell, by positioning only one LED and setting the boundary surfaces to be perfect mirrors, as illustrated in Fig. 12. The bottom of the silicone glue is fully covered with white scattering paint of $98 \%$ reflectivity. The optical performance overview of the model without and with collimator filter mounted on was simulated and is shown in Fig. 13 (c). The luminous intensity distribution without filter displays a good Lambertian pattern at the far field. The one with the collimator filter showed a collimated beam with a FWHM of $36^{\circ}$. According to the simulations, the luminous intensity along the normal increases more than threefold for these choices of dimensions and reflectivity. The true colour intensity distribution in Fig. 13 (a) and (b) proves the beam collimating effect of the designed model.

Based on the optical performance prediction from ray tracing simulation, a testing prototype of the large area planar collimator was fabricated, as displayed in Fig. 14(a). The filter glass lies on top and optically in contact with the 4-mm thick, 50x50mm square dimension silicone rubber with nine LEDs irregularly located inside. The LED light is emitted from the top surface, while the other fives surfaces are covered with highly reflective white paint to achieve light recycling. At first, the light distribution patterns were directly observed on a white diffusive screen perpendicular to the sample plane, without and with collimator filter respectively given in Fig. 14(c) and (d). Clearly it can be seen that without the filter, light gives out a uniform blue Lambertian emission. The radiation has a collimated beam profile when the filter is applied. Greenish light leakage with low brightness was measured at large output angle from $70^{\circ}$ to $90^{\circ}$, which was also predicted with the LightTools simulation of Fig. 13(b).

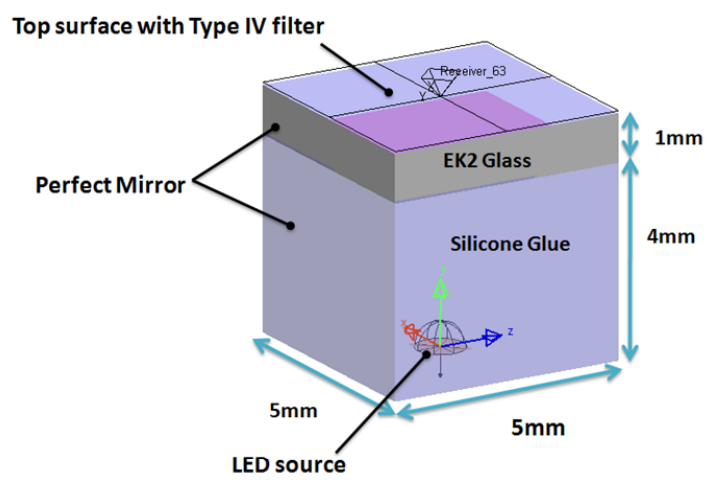

Fig. 12: Unit cell model of the planar collimator in LightTools 


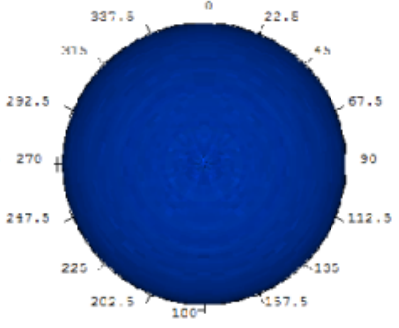

(a) without filter

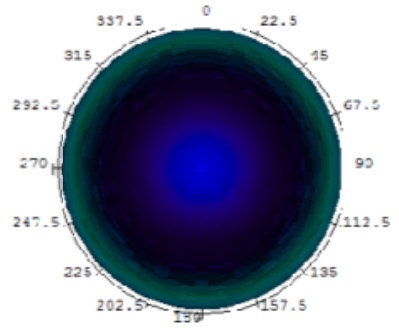

(b) with filter

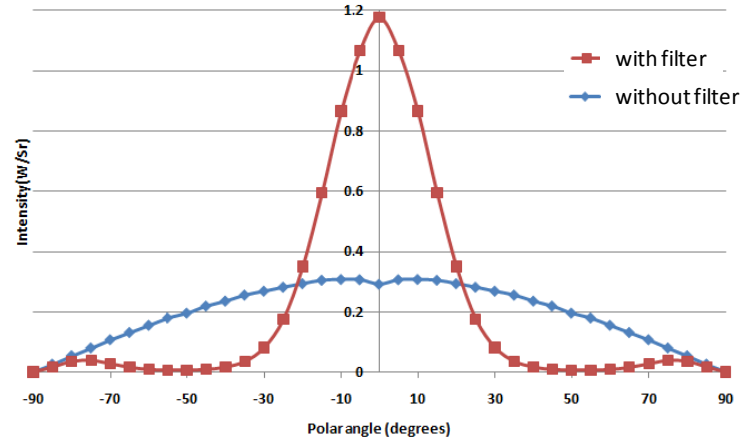

(c) cross sections

Fig. 13: Simulated true color luminous intensity of the far field angular distribution of the planar collimator

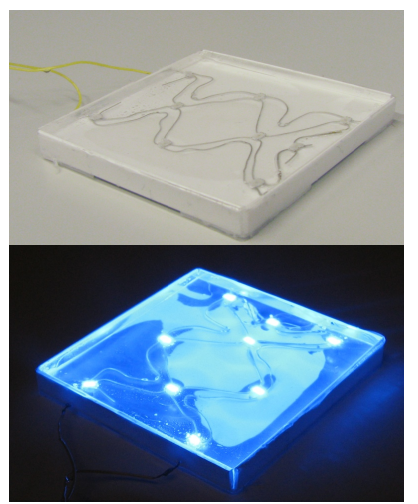

(a) without filter

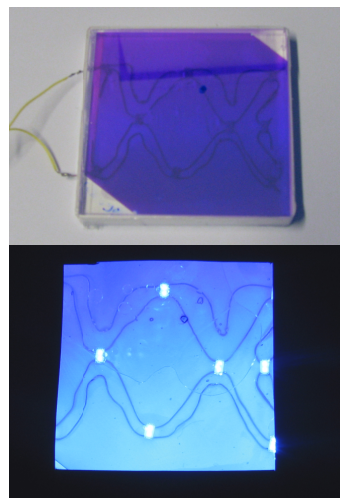

(b) with filter

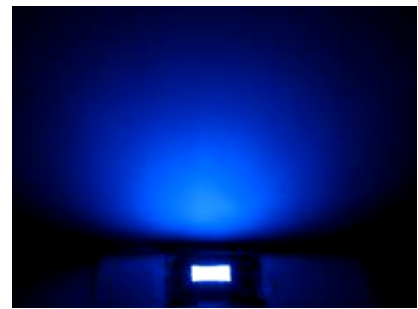

(c) without filter

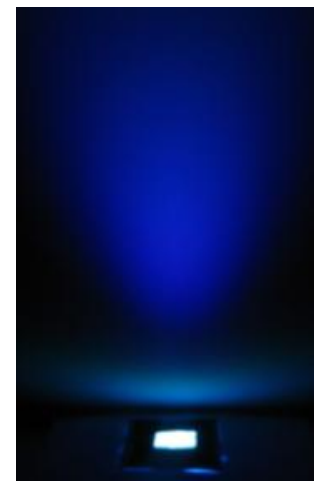

(d) with filter

Fig. 14: $(a, b)$ Pictures of the planar collimator prototype; $(c, d)$ the radiation luminous projection pattern.

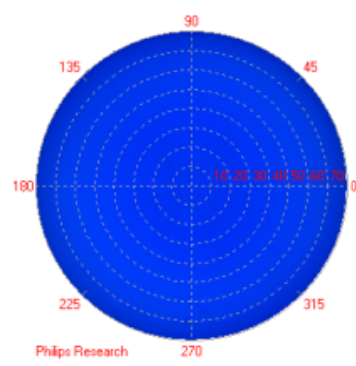

(a) without filter

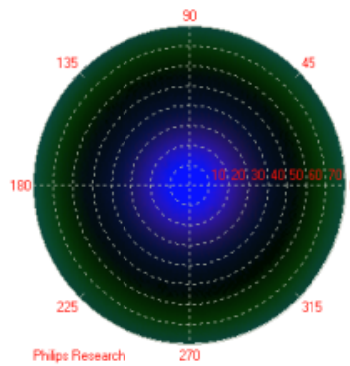

(b) with filter

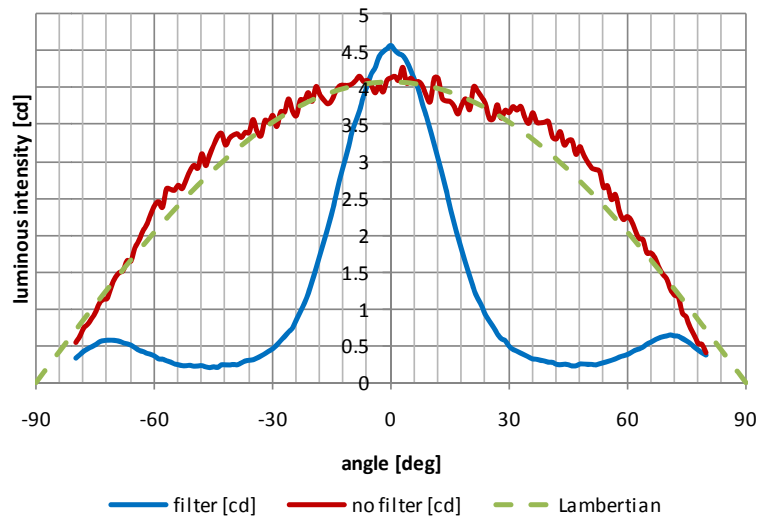

(c) cross sections of the luminous intensity

Fig. 15: Measured angular characteristics of the luminance distribution of a small part of the planar collimator prototype

The luminance distribution for angles from $-80^{\circ}$ to $+80^{\circ}$ of a $4 \mathrm{~mm}^{2}$ area in-between the LEDs was measured with a conoscope EZContrast160R from company ELDIM, shown in Fig. 15 both without filter and with filter mounted on. It 
can be seen that light with FWHM of $36^{\circ}$ is obtained, including a greenish ring at larger angle. This is in good agreement with the simulation results of Fig. 13. Further quantitative comparison between simulations and this experiment is not possible, because of the small field of view of the conoscope and the random positioning of the LEDs inside the sample.

Spectra and efficiency of the planar collimator sample were measured in an integrating sphere set-up. Fig. 16(a) indicates the normalized radiant spectra with and without filter attached. It can be seen that for this LED type the spectrum shifts $4 \mathrm{~nm}$ to shorter wavelengths when the driving current increases from $5 \mathrm{~mA}$ to $60 \mathrm{~mA}$. The addition of the filter shifts the peak of the spectrum even further because it blocks the longer wavelengths. The efficiency, defined as the ratio of the measured flux with and without the filter is increasing with driving current as shown in Fig. 16(b). It has a dependency on peak wavelength, from $21.8 \%$ to $27.7 \%(471.5 \mathrm{~nm}$ to $468 \mathrm{~nm})$. The efficiency and the normal luminous intensity can be enhanced by using white paint with higher reflectivity, seen from the calculated intensity performance in Fig. 17(a). Some light absorption of the $\mathrm{Ta}_{2} \mathrm{O}_{5}$ material in the collimator filter also reduces the total efficiency. The LightTools calculated efficiency in Fig.17 (b) increases with increasing driving current (5mA to $60 \mathrm{~mA})$ and decreasing peak wavelength (471.5nm to $468 \mathrm{~nm}$ ), which behaves similarly to the measured results shown in Fig. 16(b).

Beneficially, the LED positions are not fixed and the distance between one another is inconstant and flexible. The planar collimator system can be simply manufactured without any precise alignment and complicated shaped reflecting coating.

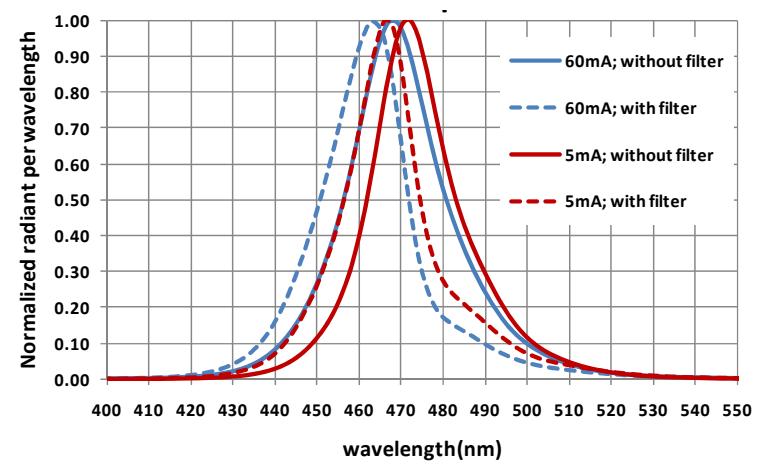

(a)
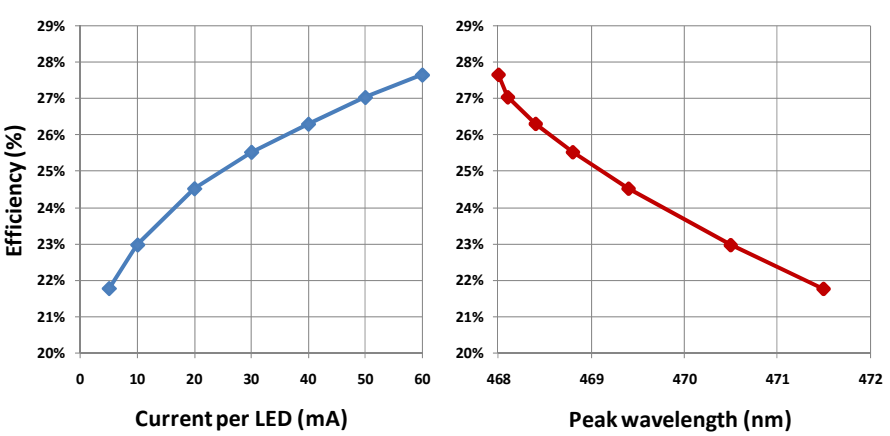

(b)

Fig. 16: (a) Measured normalized radiant spectra of the planar collimator. Blue for $60 \mathrm{~mA}$, red for 5mA; solid without filter, dashed with filter. (b) Measured efficiency at different driving current per LED and various peak wavelengths.

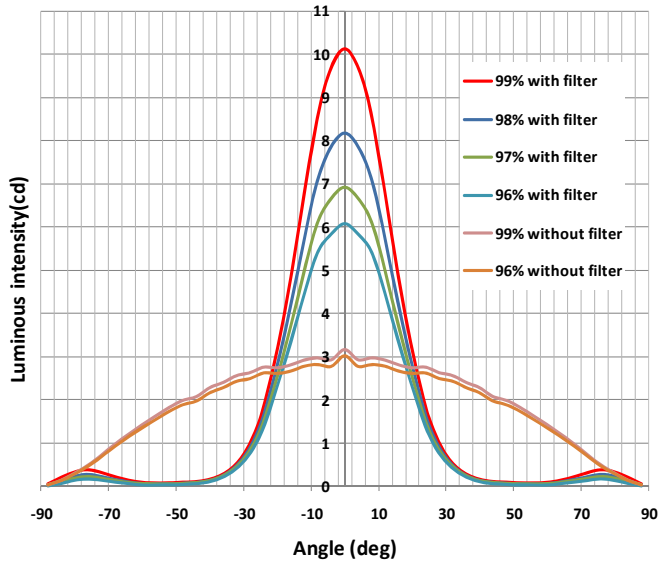

(a)
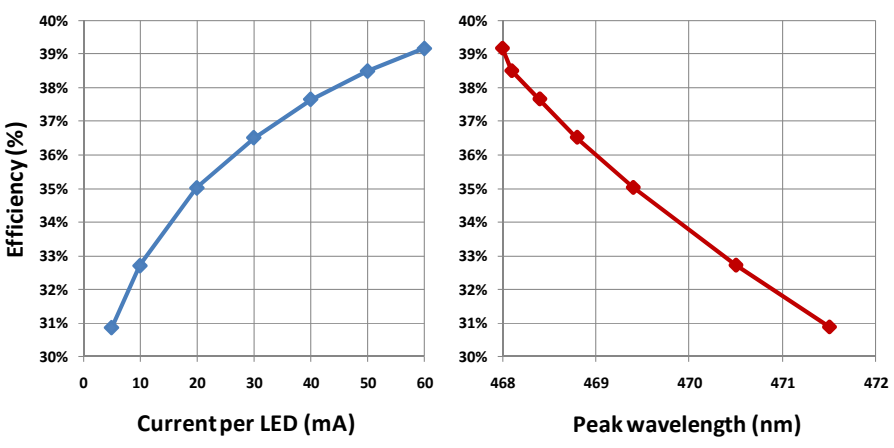

(b)

Fig. 17: (a) Calculated luminous intensity at a peak wavelength of $468 \mathrm{~nm} v s$ white paint with various reflectivity; (b) Calculated efficiency for a reflectivity of $98 \%$ at different driving current per LED and various peak wavelength. 


\section{CONCLUSION}

We have developed a new approach to achieve large-area LED luminaires, using a dielectric multilayer as an angular filter for beam shaping. A first type of filter reflects light for small angles of incidence, thus masking the LEDs and enhancing spatial spreading over large areas by total internal reflection of the LED light. This filter also enables small and compact collimators. Improvements of polarization equality for this LED-masking filter are obtained when applying polymeric material-, birefringent material- or HML material multilayers. A second type of filter complementarily transmits light for small angles of incidence, thus enabling a novel large area planar collimated light source. We see good agreement between our simulation results and measurements on prototypes. The whole planar collimator system is scalable, simply by increasing the number of embedded LEDs and/or the size of the light guide. A new approach to thin film filter design, the so called saddle-point method of global optimization was explored and it shows promising initial results.

\section{REFERENCES}

[1] Serge Bierhuizen, Michael Krames, Gerard Harbers, Gon Weijers, "Performance and trends of high power Light Emitting Diodes", SPIE 6669, 66690B (2007).

[2] R. S. West, H. Konijn, W. Sillevis-Smitt, S. Kuppens, N. Pfeffer, Y. Martynov, Y. Takaaki, S. Eberle, G. Harbers, "High brightness direct LED backlight for LCD-TV", SID 03 Digest, 1262 (2003).

[3] C.H. Ho, H.J. Cornelissen, M.P.C.M. Krijn, and H.A. van Sprang, "Multilayer-Incoupling-Structure Design for Ultra-Thin LED-Backlights", International Display Workshop 2008 IDW08, 757-760 (2008).

[4] Timothy J. Nevitt, Timothy J. Hebrink, Michael F. Weber, "Thin hollow backlights with beneficial design characteristics", US 2010/0136953 A1, (2010).

[5] John A. Wheatley, Gilles J. Benoit, James E. Anderson, Rolf W. Biernath, David G. Freier, Thomas R. Hoffend, C. David Hoyle, Tao T. Liu, Jenna D. Lu, Michael A. Meis, Vadim V. Savvateev, Craig R. Schardt, Matthew E. Sousa, Michael F. Weber, and Timothy J. Nevitt, "Efficient LED light distribution cavities using low loss, angle-selective interference transflectors", Optics Express 17, Issue 13, 10612-10622 (2009).

[6] Michael F. Weber, Carl A. Stover, "Giant Birefringent Optics in Multilayer Polymer Mirrors", Science 287, 2451$2456(2000)$.

[7] Hugo J. Cornelissen, Chenhung Ho, Haiyan Ma, Marcel P.C.M. Krijn, Hans A. van Sprang "Injecting light of high power LEDs into Thin Light Guides", Proc. of SPIE 7652, 765212 (2010).

[8] F. Bociort, E. van Driel, and A. Serebriakov, "Network structure of the set of local minima in optical system optimization", Proc. SPIE 5174, 26-34 (2003).

[9] O. Marinescu, and F. Bociort, "Network search method in the design of EUV lithographic objectives", Applied Optics 46, 8385-8393 (2007).

[10]H. Gross, H. Zuegge, M. Peschka, F. Blechinger, "The Saddle Point Method of Bociort”, Handbook of Optical Systems 3, Wiley-VCH, 362-365 (2007). 